\title{
L’Aïeul maternel de Sir Georges-Étienne Cartier - Joseph Paradis (1732-1802), pionnier de Saint-Antoine-sur-Richelieu
}

Jean Jacques Lefebvre

Volume 14, numéro 3, décembre 1960

URI : https://id.erudit.org/iderudit/302075ar

DOI : https://doi.org/10.7202/302075ar

Aller au sommaire du numéro

Éditeur(s)

Institut d'histoire de l'Amérique française

ISSN

0035-2357 (imprimé)

1492-1383 (numérique)

Découvrir la revue

Citer ce document

Lefebvre, J. J. (1960). L'Aïeul maternel de Sir Georges-Étienne Cartier — Joseph Paradis (1732-1802), pionnier de Saint-Antoine-sur-Richelieu. Revue d'histoire de l'Amérique française, 14(3), 472-474. https://doi.org/10.7202/302075ar d'utilisation que vous pouvez consulter en ligne. 


\section{BIBLIOGRAPHIE}

\section{L'AIIEUL MATERNEL DE SIR GEORGES-ETTIENNE CARTIER JOSEPH PARADIS (1732-1802), PIONNIER DE SAINT-ANTOINE-SUR-RICHELIEU}

En sa biographie, plutôt anecdotique, de sir Georges-Etienne Cartier, dont il avait été le secrétaire, Benjamin Sulte décrit l'existence aisée que connurent les paroisses du Richelieu aux lendemains de 1763 , quand pour la première fois les Canadiens se mirent à récolter assez de céréales pour en exporter.

Des marchands parsemés ici et là dans les villages y connurent, sinon une large aisance, du moins une vie confortable.

Aux premiers registres de la paroisse Saint-Antoine-surRichelieu, que devait illustrer, par sa naissance (1814), l'un des auteurs de la constitution de 1867, sir Georges-Etienne Cartier, apparaît assez souvent entre 1752 et 1801 la très belle signature de Joseph Paradis, négociant. Par le mariage, en 1798, de sa fille Marguerite P., (1778-1848) au lieutenantcolonel Jacques Cartier, ${ }^{1}$ il est connu qu'il était le grand-père du futur homme d'Etat.

Mais qui était exactement Joseph Paradis ? Il a fallu plus d'un recoupement pour établir son identité certaine, et encore davantage pour retrouver le patronyme véritable de la mère de Marguerite Paradis, née Marie-Josephte Léveillé.

Fils d'un Rochelois, François-Joseph Paradis (1700-1739), qui avait épousé à Québec, ${ }^{2}$ en octobre 1727 , Véronique Constantin ( $f l$. 1702-1751) - fille de Denis C., et de Barbe Bélanger Joseph Paradis, né à Québec le 10 juillet 1732, avait à peine 18 ans quand il contracta, en sa ville natale, au mois d'août 1750, un premier mariage avec Geneviève Marchessaut (1726-1764), née à Québec, décédée à Saint-Antoine.

Deux ans après son mariage, Joseph Paradis était déjà établi comme marchand - il avait vingt ans - à Saint-Antoine, où lui naquirent ses enfants, entre autres, Louise, en 1752, mariée a) à Louis-Frederick Blankford; b) à Belœil en 1778 à Zacharie Hurtubise (veuf de Marguerite Barsalou) et décédée à Montréal en 1780 ; et Joseph-Charles, en 1757. Ce dernier devait aller

1 C. Tanguay, Dictionnaire ... II : 512.

2 C. Tanguay, Dictionnaire ... VI: 225, 227, 229. 
prendre femme - Marie-Joseph Migneron - à Québec en 1783, mais il revint s'établir à Saint-Antoine. Il y mourut, encore jeune, en 1805.

Aux derniers jours de la Nouvelle-France, Joseph Paradis était associé avec le sieur Boileau, de Chambly - très probablement Pierre Boileau (1716-1783), plus tard capitaine de milice, le père de René Boileau, député de Chambly au I ${ }^{\text {er }}$ Parlement en 1792, pour le transport des effets du Roi, de Sorel au fort SaintJean. ${ }^{3}$ Au règlement de comptes de 1763 , il était assez riche pour présenter des lettres de change au montant de plus de 83.000 livres. ${ }^{4}$ N'y était-il qu'un prête-nom, comme, par exemple, d'Hertel de Rouville, l'ancien commandant du fort Chambly ? ${ }^{5}$ Fort probable. Il n'en dut tout de même récolter, comme la plupart des créanciers de l'époque, que le quart ou le cinquième de la valeur.

La femme de Joseph Paradis, Geneviève Marchessaut, mourut en couches, en septembre 1764. Elle n'était âgée que de 38 ans.

\section{LE CAPITAINE CHRISTOPHE MARCHESSAUT}

Elle avait, entre autres, un neveu, Christophe Marchessaut (1744-1814), né à Québec - fils de J.-B., et de Jeanne Corbin $(\dagger 1787$, Saint-Antoine) - homme relativement instruit pour l'époque, et qui devint l'un des premiers capitaines de milice de Saint-Antoine, où il se maria trois fois :

a) en 1770, à Françoise Guertin ( $\uparrow 1777)$;

b) en 1778, à Thérèse Larue (†1804)—dont la fille, Thérèse, épousa en 1805, Pierre-Antoine Gauthier ( $f l .1780-1843$ ), notaire (1803-1843) de Boucherville - fils de Pierre Gauthier (17521822) aussi notaire (1789), d'abord à Saint-Antoine, puis à Boucherville;

c) enfin, en 1805, à Anne Garant, dont un fils, Godefroy (1811-1881), ordonné en 1834, fut curé de Sainte-Rosalie, 1842, et de Saint-Aimé en 1872, et un autre, Clément, épousa en 1831, Rosollie Gravel, née en 1809, sœur de Messire Isidore Gravel (1816-1881) curé d'Iberville en 1843, de Laprairie (1854-1877), et demi-sour, entre autres, de Mgr Elphège Gravel (1838-1908) premier évêque de Nicolet (1885) et de Malvina Gravel, née en 1847, mariée en 1865 à Me Aimé Geoffrion, notaire (18651905) de Verchères.

3 P.-G. Roy, Bigot et sa bande, (Lévis, 1950), 217, 218.

4 Rapport de l'Archiviste de la P. de Q., (1924-25), 267.

5 P.-G. Roy, op. cit., 216. 
Le capitaine Christophe Marchessaut mourut à SaintAntoine en 1814.

En novembre $1766,{ }^{6}$ après contrat de mariage devant J.-C. Panet, Joseph Paradis convolait, à Québec, avec Marie-Josephte Léveillé.

Restée jusqu'ici imparfaitement identifiée, elle était née Billy-Léveillé, fille de François Billy ${ }^{7}$ ( $\left.f l .1716-1753\right)$ et de Marie-Josephte Chamard.

Sa sœur, Elisabeth, à son mariage avec Jacques Gaudry, célébré à Saint-Antoine, en 1775 , est déclarée avoir pour témoin son beau-frère, Joseph Paradis.

De sa seconde union à Marie-Josephte Léveillé, naquirent à Joseph Paradis, plusieurs enfants, nommément:

Jean-Augustin ( $f l .1767-1830$ ) - marié à Saint-Antoine en 1797 à Marie-Josephte Buron - qui s'établit négociant à SaintMarc;

Justinien (1782-1809) décédé à Saint-Marc, où il était maître d'école;

Timothée-Antoine (1785-1830) le dernier-né, qui eut pour parrain Timothy Connor; il épousa à Saint-Jean-Baptiste de Rouville, en 1812, Scholastique Chicoyne, qui lui survécut. Instituteur à Saint-Marc, comme son frère, il y mourut subitement à l'été 1830 ;

enfin, Marguerite (1778-1848) précitée - la mère de sir Georges, dont l'acte de baptême n'est pas aux registres de SaintAntoine. Peut-être fut-elle baptisée à Saint-Denis ?

Marie-Josephte Léveillé-Paradis s'éteignit en sa $52^{\mathrm{e}}$ année, à Saint-Antoine, en avril 1796.

Le 30 janvier 1801, Joseph Paradis était parrain, à SaintAntoine, avec la tante de l'enfant, Geneviève Cartier (17621811), de son petit-fils, Joseph-Gédéon Cartier - fils de Jacques $C$., et de Marguerite Paradis. C'est la dernière fois que la belle signature de Joseph Paradis apparaît aux registres de la paroisse.

Le 20 janvier 1802, Joseph Paradis mourait tragiquement à Saint-Antoine. Il y fut inhumé le surlendemain.

JEAN JACQUES LEFEBVRE

6 P.-G. Roy, Inventaire des contrats de mariages ... de Québec, V: 59.

7 C. Tanguay, Dictionnaire ... II : 281. 